\title{
EL TRIBUNAL EUROPEO DE DERECHOS HUMANOS A LA LUZ DEL PROTOCOLO 14
}

\author{
Paula Comellas Angulo \\ Universidad Católica de Colombia
}

\section{Resumen}

El artículo está centrado en las reformas introducidas por el Protocolo 14 al Tribunal Europeo de Derechos Humanos (en adelante, TEDH), el cual entró en vigor en 2010. Con ello se pretende hacer una revisión crítica sobre dicho Protocolo y analizar sus aciertos y errores, así como la nueva configuración otorgada al TEDH. Asimismo, se estudiarán las últimas reformas introducidas por declaraciones, desarrolladas en el marco de sendas conferencias ministeriales (Interlaken, Izmir y Brighton). De esta manera, se evidenciarán algunas de las problemáticas que acechan al TEDH, sobre todo en términos de congestión y funcionamiento. En última instancia, se examinará la necesidad y pertinencia de las últimas reformas al TEDH y se concluye si fueron suficientes o si, en breve, serán necesaria nuevas reformas.

Palabras clave: derechos humanos, justicia, Derecho, Derecho Internacional.

La autora: abogada, magíster en Investigación en Ciencias Jurídicas y doctoranda en Derecho Internacional Público. Docente de Derecho Internacional Privado e investigadora el grupo de Derecho Privado y Propiedad Intelectual (GEPPI) de la Facultad de Derecho, Universidad Católica. Docente de Seminario de Investigación Dirigida en la Facultad de Derecho, Universidad de los Andes.

Recibido: 20 de marzo de 2013; evaluado: 18 de abril de 2013; aceptado: 30 de abril de 2013. 


\title{
THE EUROPEAN COURT OF HUMAN RIGHTS IN THE LIGHT OF PROTOCOL 14
}

\author{
Paula Comellas Angulo \\ Universidad Católica de Colombia
}

\begin{abstract}
This article is focused on the reforms introduced by Protocol 14 to the European Court of Human Rights (ECHR) that came into force in 2010. It is aimed to make a critical review of the Protocol and to analyze its successes and shortcomings as well as the new configuration that was given to the ECHR. Likewise we will go through the latest reforms that have been introduced by declarations developed in the context of each Ministerial Conference (Interlaken, Izmir and Brighton). Some of the problems of the ECHR will be made clear, particularly in terms of congestion and operation. In the last analysis we will examine the need and appropriateness of the latest reforms to ECHR and conclude if they were enough or if soon new reforms will be required.
\end{abstract}

Keywords: human rights, justice, Law, International Law.

About the author: Lawyer, M.S. in Legal Science Research and PhD student in Public International Law. Private International Law professor and member of the Grupo de investigaciones en derecho privado y propiedad intellectual, GEPPI (Private Law and Intellectual Property Research Group) at the Faculty of Law of the Universidad Católica. Professor of Guided Research at the Faculty of Law of the Universidad de los Andes.

Received: March 20, 2013; reviewed: April 18, 2013; accepted: April 30, 2013. 


\section{Introducción}

La Declaración Universal de Derechos Humanos, proclamada en 1948 por la Asamblea General de las Naciones Unidas, supuso el punto de partida de la protección y garantía de los derechos humanos y las libertades fundamentales. No tardaron en proliferar cuerpos legales tendientes a la protección de este tipo de derechos y, en la actualidad, contamos con una multiplicidad de órganos que no siempre garantizan la protección de los mismos.

En 1949 se creó el Consejo de Europa, con el objetivo de garantizar una unión más estrecha entre sus miembros, por medio de la protección y el desarrollo de los derechos humanos y de las libertades fundamentales. Un año después, en 1950, se desarrolló la Convención Europea de Derechos Humanos y su órgano de control, el Tribunal Europeo de Derechos Humanos (en adelante, TEDH). Desde su creación, ambos han sido objeto de numerosas reformas y modificaciones, las más importantes debidas a la adhesión de nuevos miembros al Consejo de Europa.

La elección de este tema como objeto de estudio ha venido marcada por la modificación introducida por el Protocolo 14 a la Convención Europea de Derechos Humanos, con el que se reforma el mecanismo de control del Convenio hecho en Estrasburgo en 2004. Dicho Protocolo fue abierto a la firma el 13 de mayo de 2004 y entró en vigor el 1 de junio de 2010, tras la ratificación de Rusia en febrero de ese mismo año. Este artículo se centrará en el análisis de las reformas inscritas por este Protocolo y, en consecuencia, la nueva configuración del Tribunal a la luz del mismo.

El problema jurídico que aborda esta investigación es la falencia que sufre el Tribunal en términos de funcionamiento, administración y, en particular, eficiencia. El objetivo último de este trabajo es averiguar si la reforma señalada por el Protocolo 14 ha sido suficiente o si, por el contrario, en breve se necesitará una nueva transformación para dar solución a los problemas que afronta el mecanismo internacional de protección de los derechos humanos.

La metodología está centrada en un análisis pormenorizado del Protocolo 14, teniendo en cuenta los protocolos que le precedieron. Asimismo, se examinarán las Declaraciones de Interlaken, Izmir y Brighton, mediante las cuales se define una serie de recomendaciones que apunta a nuevas reformas en el seno del TEDH. 
En primer lugar, se estudiará tanto el Consejo de Europa como el TEDH, considerando su configuración anterior a la entrada en vigor del Protocolo 14; a continuación, se revisará cada una de las reformas introducidas por dicho Protocolo, para comprobar si han sido útiles en la consecución de sus objetivos y, por último, se analizarán los propósitos de las Declaraciones de Interlaken, Izmir y Brighton, firmadas en 2010, 2011 y 2012 respectivamente.

\section{El Consejo de Europa y el Tribunal Europeo de Derechos Humanos como su órgano de control}

Tras la finalización de la Segunda Guerra Mundial, aún presentes sus terribles acontecimientos, en mayo de 1949 los líderes políticos europeos decidieron crear el Consejo de Europa. ${ }^{1}$ Se trataba de la primera institución política europea de la historia, cuya finalidad era y sigue siendo "realizar una unión más estrecha entre sus miembros para salvaguardar y promover los ideales y los principios que constituyen su patrimonio común y favorecer su progreso económico y social”. ${ }^{2}$

Como establece el Preámbulo del Estatuto, el Consejo de Europa estará compuesto por un Comité de ministros y por una Asamblea Consultiva y los miembros del Consejo serán las partes intervinientes del Estatuto. ${ }^{3}$ Desde su creación ha sido objeto de numerosas ampliaciones, y cuenta en la actualidad con 47 miembros y cinco países observadores: Canadá, Estados Unidos, Japón, México y la Santa Sede, pero el punto de inflexión del Consejo se presentó en 1989 con la entrada de nuevos Estados de Europa Central y Oriental que, después de instaurar un sistema democrático, abrieron sus puertas a este tipo de organizaciones internacionales. ${ }^{4}$

El 4 de noviembre de 1950, un año después de la creación del Consejo, fue elaborado el Convenio Europeo para la Protección de los Derechos Humanos y de las Libertades Fundamentales, lo que marcó una de las señas de su identidad: la protección de los derechos humanos. ${ }^{5}$ La firma del Convenio es requisito sine qua

Julia Ruiloba Alvariño, "El Tribunal Europeo de Derechos Humanos: organización y funcionamiento". Anuario de la Escuela de Práctica Jurídica, núm. 1 (2006): 2.

2 Consejo de Europa, Estatuto No. 001 (Londres, 5 de mayo de 1949), Art. 1.

3 Los Estados fundadores y firmantes del Estatuto de Londres en 1949 fueron Bélgica, Dinamarca, Francia, Irlanda, Italia, Luxemburgo, Noruega, Países Bajos, Reino Unido y Suecia.

$4 \quad$ Hungría en 1990, Polonia y Checoslovaquia en 1991 y Bulgaria en 1992.

5 Para una visión más concreta, se recomienda ver: José Antonio Pastor Ridruejo, Curso de Derecho Internacional Público y Organizaciones Internacionales (Madrid: Tecnos, 2010), 500-537. 
non para la adhesión al Consejo de Europa y ser parte del Consejo de Europa lo es para formar parte de la Unión Europea.

Con anterioridad a la entrada del Protocolo 11, la Convención Europea de Derechos Humanos preveía en su Título II, la creación del TEDH y de la Comisión Europea de Derechos Humanos (en adelante, la Comisión), como órganos de control y protección. El TEDH estaría formado por un número de jueces igual al de las partes contratantes y, para el examen de los asuntos que ante él se susciten, contaría con Comités formados por tres jueces, salas de siete jueces o una Gran Sala de diecisiete jueces. La Comisión estaría encargada de filtrar las demandas, de instar al arreglo amistoso, de expresar un dictamen sobre el fondo de las demandas declaradas admisibles y no resueltas por la mencionada vía amistosa, de decidir plantear el caso ante la Corte y, de hacerlo, desempeñar el papel de auxiliar de la Corte. El TEDH tendría un carácter no permanente con jurisdicción para resolver las demandas que ya habían recibido dictamen por parte de la Comisión. Además, existía la figura del Comité de ministros, el cual tenía como función resolver las demandas que, conforme al dictamen de la Comisión, no fueran sometidas al TEDH.

Por su parte, los individuos estaban autorizados a presentar sus demandas ante la Comisión, siempre y cuando el Estado demandado hubiese reconocido la competencia de esta. Por lo tanto, solo la Comisión o un Estado poseían legitimidad para someter el asunto al TEDH.

\section{Reformas anteriores al Protocolo 14}

La Convención Europea de Derechos Humanos ha sido objeto de diversas modificaciones, en ocasiones para la incorporación de nuevos derechos y, en otras, para la reforma del procedimiento y examen de las demandas por parte del TEDH. Forman parte del primer caso el Protocolo 6 al Convenio para la protección de los derechos humanos y de las libertades fundamentales, relativo a la abolición de la pena de muerte, hecho en Estrasburgo el 28 de abril de 1983.

En cuanto a las reformas sufridas por el TEDH en las cuales se variaba el procedimiento y examen de las demandas, el Protocolo 9 confirió a los individuos la posibilidad de llevar sus asuntos ante el TEDH, después de que interviniera la Comisión. Por su parte, el Protocolo 8, que entró en vigor en 1990, permitió a la extinta Comisión constituir salas en su seno y Comités de tres miembros para el examen de las 
demandas, con la intención de conseguir un funcionamiento más rápido y eficaz de la Comisión que, hasta la entrada de este Protocolo, solo se reunía en pleno.

La reestructuración más importante del TEDH se produjo con el Protocolo 11, que adquirió vigencia el 1 de noviembre de 1998. Como ya se dijo, 1989 marcó un punto de inflexión en el Consejo de Europa tras la adhesión de nuevos Estados de Europa Central y Oriental. El objetivo primordial del Consejo de realizar una unión más estrecha entre sus miembros se vio ampliado y supuso, en consecuencia, un aumento desmesurado del número de demandas que impidió al TEDH el cumplimiento de la regla de tramitar los procedimientos judiciales sin dilaciones excesivas, ${ }^{6}$ pues la cifra de demandas pasó de 404 en 1981 a 2.037 en $1993 .^{7}$

Con el fin de solucionar este problema, los Estados parte aprobaron el Protocolo 11, que introdujo una de las reformas más importantes del sistema: la fusión de la Comisión y el TEDH en un solo órgano —el TEDH—al que ahora tienen acceso directo los particulares que hayan sido víctimas de violaciones; el régimen de funcionamiento permanente del TEDH y la asignación al TEDH de la tarea de examinar la admisibilidad de las demandas, antes ejercida en exclusiva por la Comisión. Esta reforma mejoró el método de trabajo y la productividad del TEDH, pero fue insuficiente por el número de demandas que recibía. En 2002 fueron presentadas 34.700 demandas y en los nueve primeros meses de 2003, 29.650. ${ }^{8}$

Ante las insuficiencias de las reformas implantadas por el Protocolo 11, han surgido distintas sugerencias como solución al colapso por el que atraviesa el TEDH hoy en día. Una de ellas apunta a la instauración en el TEDH de los métodos de trabajo del Tribunal Supremo de los Estados Unidos. Este órgano de Estados Unidos publica una lista con los asuntos y, en virtud de su importancia, elige para su examen y sentencia, aunque sin razonar o motivar dicha elección. Esta posible solución cuenta con el apoyo del expresidente del Tribunal Europeo, Luzius Wildhaber, quien defiende que el TEDH solo debería pronunciar decisiones de principio, es decir, las que forman parte de su jurisprudencia. Otra solución plantea la regionalización del sistema actual mediante la creación de tribunales en las principales regiones de

6 Consejo de Europa, Estatuto No. 001, Art. 6, párr. 1.

7 José Antonio Pastor Ridruejo, "El Protocolo número 14 a la Convención Europea de Derechos Humanos: ¿Estamos ante la reforma que necesita el Tribunal?”, Revista Española de Derecho Internacional LVI, núm. 1 (2004): 141.

8 Pastor Ridruejo, "El Protocolo número 14 a la Convención Europea de Derechos Humanos", 142. 
Europa, manteniendo la jurisdicción superior del TEDH. Algunos expertos creen que la salida está en la conversión del TEDH a un órgano de consulta prejudicial, al que podrían solicitar opinión los tribunales nacionales de mayor instancia de cada país, en caso de duda a la hora de interpretar la Convención. Una cuarta medida propone reducir la lista de derechos y libertades. Otra, más realista, sugiere que lo mejor sería la creación de una instancia, dentro del TEDH, que se dedique en exclusiva al examen de admisibilidad de las demandas para que solo lleguen aquellas que hayan sido declaradas admisibles. También hay quienes sugieren el aumento del número de jueces y de salas. ${ }^{9}$

En 2001, el Comité de ministros creó un Grupo de Evaluación, el cual propuso dos recomendaciones en su informe final. La primera de ellas consistía en atribuir al TEDH la facultad de rehusar el examen detallado de las demandas que no conlleven ninguna cuestión sustancial respecto a la Convención. La segunda recomendación formulaba la creación de una instancia encargada del examen previo de las demandas, dentro del propio TEDH.

\section{Principales reformas introducidas por el Protocolo 14 a la Convención Europea de Derechos Humanos}

Con motivo del 50 aniversario de la Convención, los días 3 y 4 de noviembre de 2000 se celebró en Roma una conferencia europea sobre derechos humanos. En ella, los ministros de Justicia de los Estados parte adoptaron una declaración en la que manifestaban la necesidad e intención de reforma del TEDH. Tras diversas propuestas, en 2004 fue presentado un primer proyecto del Protocolo 14 que, al ser sustituido por otro, fue aprobado y abierto a la firma el 13 de mayo de 2004. Con la ratificación de Rusia, el Protocolo 14 entró en vigor el 1 de junio de 2010.

Del análisis del Preámbulo del Protocolo se pueden extraer sus razones y finalidades. En el primero de los considerandos se describe que:

[...] es necesario y urgente enmendar ciertas disposiciones de la Convención a fin de mantener y reforzar la eficacia a largo plazo del sistema de control en razón principalmente al aumento continuo de la carga de trabajo del TEDH y del Comité de Ministros del Consejo de Europa.

Pastor Ridruejo, "El Protocolo número 14 a la Convención Europea de Derechos Humanos", 142-143. 
Por su parte, en el segundo considerando se declara: "es necesario velar porque el Tribunal continúe desempeñando su papel preeminente en la protección de los derechos humanos en Europa". Las reformas llevadas a cabo por este Protocolo tienen dos objetivos primordiales: aumentar la productividad del TEDH para asegurar su eficacia, evitar tramitaciones dilatadas y asegurar la ejecución de las sentencias y la independencia de los jueces en el ejercicio de sus funciones. ${ }^{10}$

La reforma establecida en los Artículos 26 y 27 tiende a la consecución de ese primer objetivo, asignando al juez único funciones jurisdiccionales. El Artículo 26.1 señala:

Para el examen de los asuntos que se le sometan, el Tribunal actuará en formación de juez único, en Comités compuestos por tres jueces, en Salas de siete jueces y en una Gran Sala de diecisiete jueces. Las Salas del Tribunal constituirán los Comités por un periodo determinado.

Las competencias de este juez único aparecen recogidas en el nuevo Artículo 27, entre las que destaca, en su párrafo primero, la posibilidad del juez único de declarar inadmisible o archivar de manera definitiva una demanda individual, de conformidad con el Artículo $34^{11}$ y cuando tal decisión pueda adoptarse sin necesidad de proceder a un examen complementario. En ese caso, el juez único remitirá la demanda a un Comité de tres jueces o a una Sala. ${ }^{12}$ A juicio de Pastor Ridruejo, a pesar de considerar al juez único como personal altamente cualificado,

10 "Ante esta enorme carga de trabajo y la preocupación porque el sistema siga siendo sostenible, comenzó la búsqueda de soluciones. En 2004 se acordaron diversas medidas incluyendo la adopción del Protocolo n ${ }^{\circ}$ 14, diseñado específicamente para permitir al Tribunal tratar ciertos casos con mayor rapidez, reduciendo en particular el tiempo dedicado a los manifiestamente improcedentes o repetitivo. Aunque se estima que con su entrada en vigor la productividad del Tribunal aumentará en torno a un 20\%, los expertos coinciden en que no será suficiente para resolver el problema". Elsa García-Maltrás de Blas, "Dilaciones indebidas y duración de los procesos en el Consejo de Europa y el Tribunal Europeo de Derechos Humanos: del tiempo razonable al tiempo óptimo y previsible", Indret, Revista para el Análisis del Derecho 2, núm. 2 (abril 2007): 5.

11 "El Tribunal podrá conocer de una demanda presentada por cualquier persona física, organización no gubernamental o grupo de particulares que se considere víctima de una violación, por una de las Altas Partes Contratantes, de los derechos reconocidos en el Convenio o sus Protocolos. Las Altas Partes Contratantes se comprometen a no poner traba alguna al ejercicio eficaz de este derecho". Consejo de Europa, Protocolo número 14 al Convenio para protección de los derechos humanos y de las libertades fundamentales, por el que se modifica el mecanismo de control de convenio (Estrasburgo, 13 de mayo de 2004), Art. 34.

12 "1. El juez único podrá declarar inadmisible o eliminar del registro de asuntos del Tribunal una demanda presentada en virtud del artículo 34, cuando pueda adoptarse tal resolución sin tener que proceder a un examen complementario.

2. La resolución será definitiva.

3. Si el juez único no declara inadmisible una demanda ni la elimina del registro de asuntos, dicho juez remitirá la misma a un Comité o a una Sala para su examen complementario". Consejo de Europa, Protocolo número 14, Art. 27. 
"una decisión definitiva de inadmisibilidad o archivo ofrece mayores garantías de acierto si es adoptada por un cuerpo colegiado (Comité de tres jueces o Sala)". ${ }^{13}$

Cabe destacar que, debido a la aparición de los jueces únicos, se prevé la creación de una nueva figura: la de los relatores (rapporteurs), definida en el Artículo 24.2, ${ }^{14}$ quienes forman parte de la Secretaría y cuya función principal es clasificar las demandas para su posterior remisión al juez único.

El Artículo 35, en su párrafo 3, implanta una nueva causa de inadmisibilidad de las demandas individuales presentadas en virtud del Artículo $34,{ }^{15}$ si considera que:

[...] el demandante no ha sufrido un perjuicio importante, a menos que el respeto de los derechos humanos garantizados por el Convenio y por sus Protocolos exija un examen del fondo de la demanda, y con la condición de que no podrá rechazarse por este motivo ningún asunto que no haya sido debidamente examinado por un Tribunal nacional.

El problema ante esta reforma es de carácter conceptual, ya que el Protocolo no señala en qué circunstancias un perjuicio podrá ser considerado importante y en cuáles no.

Otro de los artículos reformados por el Protocolo 14 es el nuevo Artículo 28, el cual hace referencia a la competencia de los Comités. Por un lado, se los faculta, al igual que al juez único, a declarar inadmisible y eliminar una demanda cuando pueda adoptarse, sin necesidad de recurrir a un examen complementario. Por otro, pueden declarar la admisibilidad de una demanda y a la vez dictar sentencia sobre el fondo, si la cuestión que subyace, "relativa a la interpretación o la aplicación del Convenio o de sus Protocolos, ya ha dado lugar a jurisprudencia bien establecida del Tribunal". En ambos casos, las resoluciones y sentencias dictadas tendrán carácter definitivo.

El Artículo 29 también ha sufrido modificaciones y el nuevo precepto indica que si el juez único o el Comité no adopta la resolución en virtud de los Artículos 27

13 Pastor Ridruejo, "El Protocolo número 14 a la Convención Europea de Derechos Humanos", 144.

14 "Cuando esté constituido en formación de juez único, el Tribunal estará asistido de ponentes, que actuarán bajo la autoridad del Presidente del Tribunal. Formarán parte de la Secretaría del Tribunal". Consejo de Europa, Protocolo número 14, Art. 24.2.

15 Las condiciones de admisibilidad pueden verse en el Artículo 35 del Protocolo. 
y 28, "una Sala se pronunciará sobre la admisibilidad y el fondo de las demandas individuales presentadas en virtud del artículo 34".

Todas las reformas contenidas en los anteriores Artículos tienden a la consecución del primer objetivo del Protocolo 14: aumento de la productividad del TEDH, para conseguir una mayor eficacia y tramitación de las demandas en un lapso prudencial. Sin embargo, existen otros artículos que, a pesar de que no guardan relación con este objetivo, también han introducido reformas y por ello son merecedores de atención.

El primero es el nuevo Artículo 23 y señala que el mandato de los jueces tendrá una duración de nueve años y que no serán reelegibles. Además, fija un límite de edad de 70 años e imposibilita el ejercicio pasada dicha edad. En caso de ser sustituidos, permanecerán en sus funciones hasta ese momento y continuarán con el conocimiento de los asuntos ya asignados. En su apartado 3 introduce una novedad tendiente a la consecución del segundo objetivo: la garantía de independencia de los magistrados en el ejercicio de sus funciones. Así, para que un juez sea relevado de sus funciones será necesaria una mayoría de dos tercios del resto de los jueces y que este haya dejado de reunir las condiciones necesarias para serlo.

Otra innovación importante es señalada por el párrafo 3 del Artículo 36. El comisario de Derechos Humanos del Consejo de Europa podrá presentar observaciones por escrito y participar en la vista de cualquier asunto que se suscite ante una Sala o ante la Gran Sala.

A pesar del gran número de reformas introducidas por el Protocolo 14, faltan algunas otras que aporten coherencia a la relación entre la Carta de los Derechos Fundamentales de la Unión Europea y el Convenio Europeo de Derechos Humanos.

El Artículo 52 de la Carta de Derechos Humanos de la Unión Europea persigue, en su Artículo 3, la garantía del vínculo entre ambos textos legales. Si los derechos recogidos en la Carta se corresponden con los del Convenio, su sentido y alcance serán iguales y podrán determinarse tanto por la Carta como por el Convenio y por la jurisprudencia del TEDH y del Tribunal de Justicia. ${ }^{16}$

16 Carmen Morte Gómez, "El Convenio Europeo de Derechos Humanos: primeros pasos para una nueva reforma", Anuario de Derechos Humanos. Nueva Época 13, núm. 1 (2004): 756-757. 


\section{Últimas reformas dirigidas a agilizar el funcionamiento del Tribunal Europeo de Derechos Humanos}

Como se ha evidenciado, el principal problema del TEDH hoy en día es la congestión de demandas, lo cual afecta su funcionamiento y reputación. A finales del año 2011 aún estaban pendientes de tramitación 151.624 asuntos ante el TEDH. ${ }^{17}$ Cabe destacar que, de los 47 países miembros del TEDH, son 6 los que protagonizan estos asuntos, entre ellos, Rusia (30\%), Rumania, Polonia, Turquía, Italia y Ucrania.

En aras de mejorar esta situación, se celebraron tres conferencias con sus respectivas declaraciones: Interlaken, Izmir y Brighton, en 2010, 2011 y 2012 respectivamente. A continuación se analizará cada una de sus declaraciones hasta llegar a las últimas reformas propuestas.

\section{a. Declaración de Interlaken}

Esta conferencia, de carácter ministerial, tuvo lugar el 18 y 19 de febrero de 2010 en el seno de la Presidencia suiza del Comité de ministros del Consejo de Europa. El objetivo principal de esta reunión era buscar soluciones al atascamiento del TEDH, para lo cual la Declaración concluyó que los Estados debían ser garantes del cumplimiento del Convenio con el fin de descargar de asuntos al TEDH. ${ }^{18}$

Esta Declaración consagra una serie de recomendaciones en pro de la descongestión del TEDH, entre las que se destaca el mandato al Comité de ministros para que diseñe un sistema de admisión de las demandas más efectivo que el de la reforma del Protocolo 14. En segundo lugar, la Declaración pone el foco de atención en la mejora de la independencia de los jueces y, sobre todo, en la necesidad de evitar que el TEDH se convierta en un juez de revisión de casos que ya han sido tratados en el ámbito nacional, lo que da lugar a la repetición de muchos casos.

No cabe duda de que por medio de esta Declaración se pusieron sobre la mesa las falencias del TEDH y, aunque no consagrase de manera formal ninguna reforma, se sentaron sus bases.

17 Estadística realizada por el TEDH. Tribunal Europeo de Derechos Humanos, "Estadísticas demandas recibidas a 2011". http://www.echr.coe.int/NR/rdonlyres/B046152B-A512-45B3- ADCC-951BD48BEF0B/0/ STATS_FR_2011.PDF (acceso marzo 23 de 2013).

18 La Declaración de Interlaken puede verse completa en http://www.eda.admin.ch/etc/medialib/downloads/ edazen/topics/europa/euroc.Par.0133.File.tmp/final_en.pdf 


\section{b. Declaración de Izmir}

Un año después de proclamarse la Declaración de Interlaken, se celebró en Izmir (Turquía) una segunda Declaración entre los días 26 y 27 de abril de 2011, esta vez bajo la Presidencia turca del Comité de ministros del Consejo de Europa. ${ }^{19} \mathrm{Al}$ igual que la primera Declaración, el objetivo principal era tratar las problemáticas del TEDH.

En cuanto a las medidas establecidas en la Declaración de Izmir, sobresale la propuesta de exigir un depósito previo a la interposición de la demanda, con el fin de reducir el número de demandas ante el TEDH. En segundo lugar, se plantea la posibilidad de instaurar un sistema de multas para quienes interpongan demandas abusivas. Por último, se debate en torno a la posibilidad de introducir la obligación de asistencia letrada desde el inicio del procedimiento ante el TEDH.

De nuevo, todos los esfuerzos de la Conferencia reflejados en la Declaración de Izmir apuntan a soluciones que mejoren el funcionamiento del TEDH.

\section{c. Declaración de Brighton}

Durante la Presidencia de Reino Unido del Comité de ministros del Consejo de Europa, el 20 de abril de 2012 tuvo lugar la Declaración de Brighton. En este caso, resalta la posición de Inglaterra con respecto al TEDH, ya que en ocasiones se niega a dar cumplimiento a las sentencias del Tribunal porque considera afectada su soberanía.

En la Declaración de Brighton se ofrece una serie de recomendaciones a corto y largo plazo. De nuevo sale a la luz el tema de la responsabilidad que le corresponde a cada Estado parte de hacer cumplir el Convenio y, de este modo, evitar que los casos lleguen hasta el TEDH y se propone la introducción de medidas garantistas en la Carta Suprema de cada uno de los ordenamientos jurídicos internos o la creación de un Defensor del Pueblo que vele por la salvaguarda de los derechos fundamentales. ${ }^{20}$

19 La Declaración de Izmir puede verse completa en http://www.echr.coe.int/NR/rdonlyres/E1256FD2-DBE541E8-B715-4DF6D922C7B6/0/20110428_Declaration_Izmir_EN.pdf

20 La Declaración de Brighton puede verse completa en http://www.echr.coe.int/NR/rdonlyres/8AC14EA9A92B-4875-A76A-4E21A8B3AC5A/0/ENG_20120418_BRIGHTON_DECLARATION_FINALE.pdf 
En segundo lugar, se trató el principio de la subsidiariedad como solución factible a la congestión actual del TEDH. Este principio, consagrado en el Artículo 1 de la Convención, ya había sido introducido en la reforma llevada a cabo por el Protocolo 14, aunque no de manera explícita, por lo que la Declaración propone modificar directamente el Artículo 1 de la Convención. ${ }^{21}$

Al igual que en las Declaraciones de Interlaken e Izmir, se trata el rol del TEDH como un tribunal de casación, para evitar que se convierta en un tribunal de cuarta instancia. Al respecto, se propone revisar los criterios de admisión de las demandas y mejorar los canales de comunicación entre el TEDH y los tribunales nacionales de los Estados miembros.

Sin lugar a dudas, el TEDH necesita una nueva reforma, con el fin de implementar medidas concretas que solucionen las problemáticas de este Tribunal. Quizá en breve seamos testigos de esas nuevas reformas.

\section{Conclusiones}

La última reforma a la que ha sido sometido el TEDH resulta insuficiente a la luz del Protocolo 14. Las modificaciones introducidas no garantizan que el Tribunal vaya a superar la asfixia que hoy le agobia, objetivo perseguido por el Protocolo 14. El número de jueces no ha variado en ningún momento y lo único que se ha producido es una redistribución de la carga de trabajo mediante la transferencia de funciones de órganos ya establecidos a otros nuevos de formación más reducida (juez único). Por otra parte, el aumento del número de jueces solo se plantea para el caso de nuevas adhesiones. Si se cree que el número de demandas también aumentaría, resulta del todo escaso.

Al considerar el volumen de demandas pendientes ante el TEDH y la demora en su tramitación y ejecución, el Protocolo 14 debió proponer una reforma más profunda y valiente. Debían haberse tenido en cuenta algunas de las soluciones planteadas por el Grupo de Evaluación creado por el Comité de ministros en 2001, es decir, atribuir al Tribunal la facultad de rehusar el examen detallado de las demandas que no planteen ninguna cuestión sustancial respecto a la Convención o crear dentro del propio Tribunal una instancia encargada del examen previo de las demandas.

21 Fernando Irurzun Montoro, “¿Una nueva reforma del Tribunal Europeo de Derechos Humanos?”, Papeles de Derecho europeo e integración regional, núm. 10 (2012): 5-6. 
En aras de la descongestión del TEDH, el Protocolo 14 podría haber introducido alguna reforma tendiente al cumplimiento del principio de subsidiariedad y del deber de todo Estado miembro de instaurar en sus sistemas nacionales los recursos eficaces para reparar las violaciones de la Convención, recogidos en sus Artículos 1 y 13, respectivamente. Según el principio de subsidiariedad, el TEDH actúa solo en el caso de que los Estados fallen en su misión de respeto y garantía de los derechos humanos y declarará admisible una demanda cuando se hayan agotado las vías definidas por cada Estado para ello. De tal manera, si cada Estado cumpliera con la obligación de instaurar recursos eficaces en sus sistemas, los individuos se verían menos obligados a recurrir al TEDH.

Las Declaraciones de Interlaken, Izmir y Brighton evidencian las carencias del TEDH en términos de organización y funcionamiento. En estas Declaraciones se recogen recomendaciones concretas que podrían acabar con dichas problemáticas, por lo que en breve podría y tendría que hacerse una nueva reforma al Tribunal.

Como órgano de control, el TEDH constituye uno de los instrumentos más valiosos del Derecho Internacional. Por ello, debe hacerse todo lo posible para su salvaguarda y, sobre todo, para la consecución de un funcionamiento eficaz como órgano de protección y garantía de los derechos humanos y de las libertades fundamentales.

\section{Referencias}

Consejo de Europa. Convención para la protección de los derechos humanos y las libertades fundamentales. Roma, 4 de octubre de 1950.

Consejo de Europa. Estatuto No. 001. Londres, 5 de mayo de 1949.

Consejo de Europa. Protocolo número 11 al Convenio para protección de los derechos humanos $y$ de las libertades fundamentales relativo a la reestructuración del mecanismo de control establecido por el Convenio. Estrasburgo, 11 de mayo de 1994.

Consejo de Europa. Protocolo número 14 al Convenio para protección de los derechos humanos y de las libertades fundamentales, por el que se modifica el mecanismo de control de convenio. Estrasburgo, 13 de mayo de 2004.

Consejo de Europa. Protocolo número 6 al Convenio para protección de los derechos humanos y de las libertades fundamentales, relativo a la abolición de la pena de muerte. Estrasburgo, 28 de abril de 1983.

Consejo de Europa. Protocolo número 8 al Convenio para protección de los derechos humanos y de las libertades fundamentales. Viena, 19 de marzo de 1985. 
Consejo de Europa. Protocolo número 9 al Convenio para protección de los derechos humanos y de las libertades fundamentales. Roma, 6 de noviembre de 1990.

García-Maltrás de Blas, Elsa. "Dilaciones indebidas y duración de los procesos en el Consejo de Europa y el Tribunal Europeo de Derechos Humanos: del tiempo razonable al tiempo óptimo y previsible" Indret, Revista para el Análisis del Derecho 2, núm. 2 (abril 2007): 1-20.

Irurzun Montoro, Fernando. "¿Una nueva reforma del Tribunal Europeo de Derechos Humanos?". Papeles de Derecho europeo e integración regional, núm. 10 (2012): 1-15.

Morte Gómez, Carmen. "El Convenio Europeo de Derechos Humanos: primeros pasos para una nueva reforma". Anuario de Derechos Humanos. Nueva Época 13, núm. 1 (2004): 755-784.

Pastor Ridruejo, José Antonio. "El Protocolo número 14 a la Convención Europea de Derechos Humanos: ¿Estamos ante la reforma que necesita el Tribunal?”. Revista Española de Derecho Internacional LVI, núm. 1 (2004): 141-150.

Pastor Ridruejo, José Antonio. Curso de Derecho Internacional Público y Organizaciones Internacionales. Madrid: Tecnos, 2010.

Ruiloba Alvariño, Julia. "El Tribunal Europeo de Derechos Humanos: organización y funcionamiento". Anuario de la Escuela de Práctica Jurídica, núm. 1 (2006): 1-14.

Tribunal Europeo de Derechos Humanos. "Estadísticas demandas recibidas a 2011". http:// www.echr.coe.int/NR/rdonlyres/B046152B-A512-45B3- ADCC-951BD48BEF0B/0/ STATS_FR_2011.PDF (acceso marzo 23 de 2013). 